\title{
GLOBAL ESTIMATES FOR THE MAXIMAL OPERATOR AND HOMOTOPY OPERATOR
}

\author{
YUMING XING AND SHUSEN DiNG
}

Abstract. In this paper, we develop some estimates for the Hardy-Littlewood maximal operator and the sharp maximal operator. We also establish $L^{S}$-norm inequalities related to the composite operators.

Mathematics subject classification (2010): Primary 26B10; Secondary 31B10, 46E35.

Keywords and phrases: Differential forms, harmonic equations, homotopy operator, maximal operator.

\section{REFERENCES}

[1] R. P. Agarwal And S. Ding, Advances in differential forms and the A-harmonic equations, Mathematical and Computer Modelling 37 (2003), 1393-1426.

[2] S. DING, Lipschitz and BMO norm inequalities for operators, Nonlinear Analysis 71 (2009), e2350e2357.

[3] S. Ding, Norm estimates for the maximal operator and Green's operator, The Proceeding of the 6th international conference on Differential Equations and Dynamical Systems, 2008.

[4] T. IWANIEC AND A. LUtoborski, Integral estimates for null Lagrangians, Arch. Rational Mech. Anal. 125 (1993), 25-79.

[5] B. LIU, $A_{r}^{\lambda}(\Omega)$-weighted imbedding inequalities for A-harmonic tensors, J. Math. Anal. Appl. 273 (2002), 667-676.

[6] C. A. NoldER, Hardy-Littlewood theorems for A-harmonic tensors, Illinois J. of Math. 43(1999), 613-631.

[7] C. ScotT, $L^{p}$-theory of differntial forms on manifolds, Trans. Amer. Soc 347 (1995), 2075-2096.

[8] [8] E. M. Stein, Harmonic analysis, Princeton University Press, Princeton, 1993.

[9] Y. XING, Two-weight imbedding inequalities for solutions to the A-harmonic equation, J. Math. Anal. Appl. 307 (2005), 555-564.

[10] Y. XING AND C. WU, Global weighted inequalities for operators and harmonic forms on manifolds, J. Math. Anal. Appl. 294 (2004), 294-309. 